\title{
COMMENT SE PRENNENT LES DÉCISIONS DE TRĖS GROS EQQUIPEMENTS DANS LES LABORATOIRES \\ DE « SCIENCE LOURDE » CONTEMPORAINS. UN RÉCIT SUIVI DE COMMENTAIRES
}

Un des traits de l'historiographie dominante des sciences est sa préférence pour l'étude des travaux de nature théorique, ceux du Galilée des Dialogues, du Newton des Principia, ou ceux d'Einstein en $1905^{1}$. Pour elle, le travail historique consiste principalement à élucider l'émergence des notions et concepts, à percevoir leurs déploiements, leurs enchaînements, et leurs transferts. L'aventure de l'esprit humain en lutte avec le réel l'intéresse, mais plus encore la lutte de celui-ci avec lui-même, avec les instincts de conservation de la pensée, avec les formes culturelles héritées qui l'imprègnent. Le reste - la pratique plus quotidienne de laboratoire, celle qui est censée fournir les « données expérimentales " - est traitée avec plus de désinvolture : ce qui se passe dans les arrière-cuisines de la Science n'a qu'un intérêt intellectuel limité. Un nombre assez réduit d'hommes et de textes se trouvent ainsi retenus et placés au centre du travail historique. Ils forment le matériau primaire à partir duquel le débat s'organise, à partir duquel les conceptions de ce que sont la physique ou la biologie se forment. Cette manière de procéder a comme corollaire de traiter l'activité scientifique comme essentiellement artisanale et individuelle et de tenir à l'écart aussi bien la dimension expérimentale de la pratique scientifique que celle qui vise à la construction des matériels. Le paradoxe de cette attitude, en particulier lorsqu'elle traite de la physique du dernier demi-siècle, est qu'elle tend à " oublier " qu'une part non négligeable des grands résultats théoriques a son origine dans des unités de recherche de grande taille, qui mettent en jeu des dispositifs

1. Pour des constatations similaires, voir l'avant-propos de T.S. KUHN, in Bruce R. Wheaton, The Tiger and the Shark, Empirical Roots of Wave-Particule Dualism, Cambridge, Cambridge University Press, 1983, p. xii ; Steven ShaPIN, Simon Schaffer, Leviathan and the Air-Pump, Hobbes, Boyle, and the Experimental Life, Princeton, Princeton University Press, 1985, p. 17, n. 35; Allan Franklin, The Neglect of Experiment, Cambridge, Cambridge University Press, 1986. 
lourds et coûteux, des groupes professionnels divers et qui suivent des traditions de pensée différentes, et dont les relations aux savoirs ne sont pas sans effet sur la science qui est produite.

Notre propos va être ici de regarder dans une " arrière-cuisine " de ce type en considérant la préparation d'un des ingrédients essentiels à la fabrication de la science contemporaine : la décision d'un gros équipement de recherche fondamentale. Le cas que nous avons suivi plus particulièrement est celui de la seconde génération d'accélérateurs pour le CERN, l'Organisation européenne pour la recherche nucléaire. Le CERN est un laboratoire de physique fondamentale qui fut créé au début des années 1950 par les principaux États de l'ouest de l'Europe afin d'étudier la physique dite des particules élémentaires (ou physique des hautes énergies) ${ }^{2}$. Son programme de construction, accepté en 1952, comprenait deux accélérateurs; le plus grand était un synchrotron accélérant des protons jusqu'à $25 \mathrm{GeV}$ (un synchrotron est un anneau d'aimants entourant une chambre à vide dans laquelle circulent les particules). En novembre 1959, cet anneau de 200 mètres de diamètre entrait en fonctionnement : il formait alors l'accélérateur le plus puissant au monde, une machine similaire (de $33 \mathrm{GeV}$ ) étant mise en service l'année suivante au laboratoire américain de Brookhaven, dans l'État de New York. La durée de vie de ces machines pour une recherche de pointe étant limitée (15-20 ans en première approximation) et leur construction requérant 6 à 8 ans, la question se posa, dès le début des années 1960, de prévoir leurs successeurs. C'est ce cas, ce processus de réflexion et de décision qui devait durer plus de dix ans et engager des dépenses de l'ordre du milliard de francs suisses, que nous souhaiterions considérer ici.

Nous adopterons dans un premier temps la forme du récit monographique car elle nous semble être la seule permettant de faire sentir empiriquement au lecteur les limites de certains a priori concernant la science. Suivre par le détail le processus par lequel le CERN en vint à construire certaines machines - et non d'autres - permet seul de prendre conscience de ce qu'est la fabrication d'une part de la science contemporaine. Dans un second temps, nous élargirons la réflexion. Comparant notre « roman vrai " (comme dirait Paul Veyne) à d'autres études, nous avancerons quelques conclusions sur la manière dont le débat procède entre scientifiques ainsi que quelques remarques de méthode sur le traitement historique de ces questions. Pour ce faire,

2. Armin Hermann, John Krige, Ulrike Mersits, Dominique Pestre, History of CERN, Amsterdam, North-Holland, 1987, vol. I : Launching the European Organization for Nuclear Research; pour une version abrégée, D. PESTRE, J. KRIGE, "La naissance du CERN, le comment et le pourquoi ", Relations internationales, 46, 1986, p. 209-226. 
nous ferons appel à deux études concernant l'astronomie et l'astrophysique des années 1945-1975, ainsi qu'à une série de travaux entrepris par le groupe d'étude d'histoire du CERN ${ }^{3}$.

Un dernier point : il est impossible d'être techniquement très précis dans ce texte dont nous souhaitons qu'il soit lisible par un non scientifique. Nous nous permettrons donc ici un certain nombre d'approximations que nous avons évitées dans l'étude détaillée publiée au CERN en janvier $1987^{4}$.

\section{1. - UN RÉCIT : COMMENT FUT DÉCIDÉE LA SECONDE GÉNÉRATION D'ACCELEERATEURS DU CERN}

Le choix que nous avons fait - étudier le processus de décision conduisant à la définition des accélérateurs du CERN pour les années 1970-1980 - s'appuie sur l'importance intrinsèque de l'enjeu, tant pour l'avenir de l'Europe (en physique des hautes énergies) qu'en termes financiers. Il n'aurait pourtant pas constitué un «bon » cas si nous n'avions pas disposé d'un fonds documentaire large. Dans la mesure où c'est moins le résultat en lui-même qui nous importait (tel type de machine finalement décidé) que la manière dont on y parvint, la variété comme la précision des sources était centrale. Il nous fallait, en effet, disposer non seulement de la science "publique " (revues, livres, ...), mais aussi de la science " semi publique " (la littérature dite grise) et d'une documentation privée de qualité (correspondance, en

3. David O. Edge, Michael J. MUlkay, Astronomy Transformed, the Emergence of Radio-Astronomy in Britain, New York, J. Niley \& Sons, 1976; Gérard DARMON, Psychosociologie d'une décision en science lourde, thèse 30 cycle, Paris, EHESS, 1981 ; Gérard Darmon, Gérard Lemaine, "Étude d'une décision en science lourde : le cas de l'Institut de radio-astronomie millimétrique franco-allemand", Information sur les sciences sociales, t. 21, 1982, p. 847-872. Les travaux du groupe d'histoire du CERN sont encore non publiés à l'exception de la référence, supra, n. 2 . Á propos de gros équipements en physique des hautes énergies, on lira Daniel S. Greenbero, The Politics of Pure Science, New York, Plume Book, 1971, chap. $x$ et XI; Michael GibBons, «The CERN $300 \mathrm{GeV}$ Accelerator: A Case Study in the Application of Weinberg Criteria ", Minerva, t. 8, 1970, p. 180-191 ; John L. Heilbron, Robert W. Seidel, Bruce R. Wheaton, "Lawrence and his Laboratory : Nuclear Science at Berkeley ", LBL News Magazine, t. 6, 1981, numéro spécial. Une bibliographie plus complète est dans J. KRIGE, D. PESTRE, "The choice of CERN's first large bubble chambers for the proton synchrotron (1957-1958) ", Historical Studies in the Physical and Biological Sciences, t. 16, 1986, p. 255-279.

4. D. PESTRE, La Seconde génération d'accélérateurs pour le CERN, 1956-1965. Étude historique d'un processus de décision de gros équipement en science fondamentale, Genève, CERN-CHS-19, 1987. 
particulier). Disons-le tout net : de ce point de vue, les archives du CERN furent parfaites ${ }^{5}$.

La nécessité de considérer les machines de l'après-PS de $25 \mathrm{GeV}$ se fit jour au CERN dès 1956, lorsqu'il apparut à la direction, lors d'une conférence internationale, que l'organisation était en retard en matière de recherche sur les accélérateurs par rapport aux U.S.A. et à l'U.R.S.S. ${ }^{6}$. Un groupe d'une douzaine de personnes fut donc établi, groupe qui étudia divers procédés nouveaux d'accélération. Après un an et demi d'essais, il décidait, en 1958, de construire un modèle (au sens de modèle réduit) connu sous le nom d'accélérateur bidirectionnel à électrons, de type FFAG, de $2 \times 100 \mathrm{MeV}$ d'énergie. L'idée était de faire circuler deux faisceaux en sens opposé dans la même chambre à vide (et non un seul, comme d'habitude) et donc de mettre en œuvre un type de structure magnétique sophistiquée. Ce choix répondait à une exigence simple : construire un modèle mettant en jeu le plus grand nombre possible d'idées neuves, selon le dicton, qui peut le plus, peut le moins. Ce modèle était donc d'abord un outil de recherche polyvalent?

Au cours de l'année 1960, le cadre de travail du groupe changea fondamentalement. Bien que la réalisation matérielle de l'accélérateur de $2 \times 100 \mathrm{MeV}$ fût assez avancée, le groupe en abandonna le principe et opta pour un modèle beaucoup plus simple formé d'un injecteur (commandé à une firme américaine) et d'un anneau (à construire au CERN) pour étudier l'accumulation des particules. On abandonnait donc l'idée d'un outil de recherche multidirectionnel au profit d'un outil ne visant plus qu'à tester une possibilité unique : l'accumulation des faisceaux dans un anneau de stockage. L'intérêt était l'obtention de hautes intensités dans ces anneaux ${ }^{8}$.

Le fait qu'on avait choisi de réduire le nombre des pistes de recherche apparut clairement quelques mois plus tard lorsque le groupe quitta le monde des modèles pour soumettre aux scientifiques du CERN un projet "grandeur nature " devant succéder au PS de

5. Dans la suite, les documents issus des archives du CERN seront identifiés par le seul numéro de la boîte d'archives dans laquelle ils se trouvent, soit, par exemple (DG 20576) ou (IBA 22798).

6. CERN Symposium on High Energy Accelerators and Pion Physics, Genève, CERN, 1956 ; John B. ADAMS, Research Work on Machine Design and Allied Problems, 12/10/56, rapport, CERN/SPC/33.

7. Pour une description de ce modèle et de ses motivations, M. BARBIER, et al., « Studies of an Experimental Beam-Stacking Electron Accelerator », International Conference on High Energy Accelerators and Instrumentation, Genève, CERN, 1959, p. 100-114. Le $\mathrm{GeV}$ ( $10^{9}$ électron-volt) est une unité de mesure d'énergie ; le MeV vaut $10^{6}$ électron-volt.

8. A. Sсносн, A Discussion of Colliding Beam Techniques, rapport, s.d. (en fait, automne 1960) (DG 20576). 
$25 \mathrm{GeV}$. Sa proposition était de construire deux anneaux d'accumulation devant être alimentés par des protons de $25 \mathrm{GeV}$ venant du PS (chacun d'eux étant d'une taille similaire à celle du PS lui-même) et intersectant en un (ou deux) points : les Intersecting Storage Rings (ISR). L'objectif était d'obtenir des collisions de protons se heurtant frontalement - le taux de collision était suffisant grâce aux bonnes intensités obtenues par accumulation - et d'observer en ces un ou deux points des interactions proton-proton de très haute énergie $(25$ +25 soit $50 \mathrm{GeV}$ dans le centre de masse des deux protons). Cette énergie dans le centre de masse, la seule pertinente du point de vue de la physique, était environ 7 fois plus élevée que celle obtenue lors du choc des protons de $25 \mathrm{GeV}$ sur des cibles fixes 9

Ce début de récit appelle deux questions. La première est de savoir ce qui conduisit le groupe de recherche sur les accélérateurs du CERN à changer de politique en 1960 , à passer d'un outil de recherche poly. valent à une proposition particulière de machine de grande taille. Deux raisons apparaissent nettement. La première est un changement dans le contexte international : un accord américano-soviétique commença à être négocié à la fin de 1959 pour la construction en commun d'un accélérateur géant. Les Européens en charge des accélérateurs du futur en déduisirent l'urgence, pour l'Europe de l'Ouest, de se doter elle aussi d'une nouvelle machine ${ }^{10}$. Ce sentiment se concrétisa rapidement pour une autre raison: le groupe avait doublé de taille au début de 1960, recevant notamment le renfort de quelques-uns des meilleurs spécialistes du CERN, de ceux ayant construit le PS entre 1953 et 1959 et se trouvant alors sans affectation au sein de l'organisation. Plus ingénieurs que théoriciens des machines ou physiciens, ces derniers souhaitaient reprendre rapidement un nouveau grand projet".

La seconde question serait de savoir pourquoi ces " constructeurs de machines " avancèrent l'idée particulière d'anneaux d'intersection pour le PS de $25 \mathrm{GeV}$. La réponse tient à plusieurs choses : à la tradition de recherche, à la compétence acquise dans le domaine de l'accumulation de faisceaux par le groupe de recherche sur les accélérateurs du CERN depuis 1957; au fait que ce genre d'appareillage était très novateur du point de vue de la technologie des machines; au fait que

9. H.G. Hereward, K. Johnsen, A. Schoch, C. J. Zilverschoon, Present Ideas on 25-GeV Proton Storage Rings, rapport, 22/12/60, Genève, CERN PS/Int. AR/60-35.

10. Voir les documents CERN/SPC/100, 23/9/59; CERN/CC/342/projet, 19/1/60 ; Design Study for a 300-1000 BeV Accelerator, Upton, Brookhaven National Laboratory, $28 / 8 / 61$

11. M. J. PENTZ, Accelerator Research at CERN, 1956-1967, Genève, CERN 68-9, 1967, p. 20. 
le PS de $25 \mathrm{GeV}$ se trouva fonctionner particulièrement bien (mieux que prévu), et qu'il apparut comme pouvant constituer un excellent injecteur pour d'éventuels ISR ; au fait, finalement, que ces anneaux ne pouvaient être construits qu'auprès du PS, à Genève - et donc qu'ils allaient contribuer au développement du jeune laboratoire qu'était le CERN. Précisons une chose : cette idée d'ISR fut soumise à l'ensemble du CERN comme une piste de réflexion, le groupe n'ayant pas mené une étude préalable des autres possibilités techniques ${ }^{12}$.

Le moins que l'on puisse dire est que cette proposition surprit et qu'elle fut activement débattue tout au long de 1961. Après deux ou trois mois, les positions se répartirent en deux groupes, reconnus par les acteurs eux-mêmes. D'un côté, ceux que nous avons appelé les constructeurs - ingénieurs de formation le plus souvent, responsables de tous les grands projets au CERN, ayant collaboré à la construction du PS. Ceux-ci soutinrent les ISR. De l'autre, les physiciens - utilisateurs des machines, universitaires-chercheurs le plus souvent. Ceux-là étaient en faveur de synchrotrons classiques dont le faisceau de protons peut être éjecté et envoyé sur des détecteurs extérieurs (machines du type du PS de $25 \mathrm{GeV}$, dites à cible fixe). L'argumentation majeure que les constructeurs avancèrent lorsqu'ils durent défendre le projet du groupe de recherche sur les accélérateurs fut que les ISR offraient une énergie utilisable excellente, qu'ils ouvraient une " fenêtre " sur la physique à « ultra-haute " énergie, et ce pour un prix raisonnable : un accélérateur classique, à cible fixe, et fournissant $50 \mathrm{GeV}$ dans le centre de masse aurait un rayon de plusieurs kilomètres et coûterait de l'ordre de dix fois le prix des ISR. Celle des physiciens était que les ISR ne donneraient que des interactions proton-proton à faible taux (un faisceau, même intense, n'a jamais la densité d'une cible fixe, liquide ou solide), que la géométrie de l'expérience serait imposée par la configuration de la machine (la position respective, et fixe, des deux anneaux), qu'il ne serait pas possible de créer, à partir du faisceau primaire de protons, les faisceaux secondaires de pions, kaons, antiprotons, etc., auxquels ils étaient habitués. Or, ajoutaient-ils, c'était cette flexibilité d'usage (varier les faisceaux de particules, varier les conditions expérimentales) et l'existence de ces diverses particules avec des intensités suffisantes (à défaut, les temps d'expérience peuvent être démesurément longs) qui avaient toujours fait la force des physiciens. Les ISR étaient donc le plus mauvais choix qu'on pût faire : il s'agissait moins d'un accélérateur, à leurs yeux, que d'une seule expérience

12. D. Pestre, op. cit. supra, n. 4, p. 31-35. 
proton-proton ${ }^{13}$. Remarquons ici une petite chose. Ce débat est traversé par deux logiques de raisonnement qui se rencontrent peu. Deux manières de poser la question, deux manières de définir ce qui est crucial dans le choix s'opposent globalement. Il ne peut donc vraiment y avoir un dialogue.

Réunis en conférence du 5 au 9 juin 1961, les physiciens européens confirmèrent leur position antérieure et le Comité des directives scientifiques du CERN fut appelé à trancher. Ce comité, formé de l'establishment scientifique européen extérieur au CERN, était chargé de préparer les décisions techniques à prendre par l'instance suprême de l'organisation, le Conseil des représentants des Etats. Sa conclusion fut double. Il reconnut d'abord que c'était aux futurs utilisateurs que devait revenir le dernier mot en ces matières; en conséquence, il fit sien leur projet d'accélérateur à cible fixe, d'énergie moyenne (50 à $100 \mathrm{GeV}$ ) mais de très haute intensité (100 à 1000 fois celle du PS de $25 \mathrm{GeV}$ ). Il ajouta toutefois que le choix final n'était pas à l'ordre du jour, qu'il n'y avait pas urgence. Le débat devait donc être poursuivi et aucune solution ou idée techniques ne devaient être éliminées prématurément. Pour l'instant, l'objectif était de bien utiliser le PS, de faire de la bonne physique à $25 \mathrm{GeV}^{14}$.

Durant l'été, plusieurs séminaires de travail furent tenus aux ÉtatsUnis sur l'utilisation et la technologie des accélérateurs. À leur suite, un type de machine apparut à l'énorme majorité des spécialistes comme la voie royale vers le futur, à savoir le synchrotron, à cible fixe, mais de très haute énergie (plusieurs centaines de $\mathrm{GeV}$ ). La raison majeure en était qu'il pouvait satisfaire techniquement "toutes " les exigences des physiciens. Comme l'écrivaient alors la plupart des auteurs, ces accélérateurs étaient dans leur essence même " multipurpose "; en visant l'énergie, on obtenait par-là même, intrinséquement pourrait-on dire, une excellente intensité du faisceau primaire ; parallèlement, l'énergie comme l'intensité des faisceaux secondaires se trouvaient elles aussi augmentées. Le revers de la médaille était le coût proprement prohibitif de ces machines géantes ${ }^{15}$. À l'automne, les physiciens européens (et une large part des constructeurs conscients des avantages d'utilisation de ces " monstres ») reconnurent la situa-

13. Parmi des dizaines de rapports et de lettres, voir, pour les constructeurs, M.G. N. HINE, Possible Long Term Programmes for CERN, Drafi, 30/3/61; pour les physiciens, Leon VAN HOVE, « Summary Lecture ", International Conference on Theoretical Aspects of Very High-Energy Phenomena, 5-9/6/61. Genève, CERN 61-22, 1961, p. $407-423$.

14. Le comité se réunit le 29/4/61 (compte rendu CERN/SPC/133/Draft, 7/7/61) et le 21/7/61 (CERN/SPC/140/Draft, 27/9/61).

15. Voir les conclusions de Design Study..., op. cit. supra, n. 10. 
tion nouvelle créée par ces études de l'été et un nouveau groupe de travail élargi fut constitué au CERN ${ }^{16}$. Son programme fut défini en novembre : il devait considérer en détail les paramètres techniques d'une machine de l'ordre de quelques centaines de $\mathrm{GeV}$ et poursuivre l'étude des ISR. Ces derniers n'étaient pas éliminés dans la mesure où ils avaient leurs propres avantages (notamment leur bon rapport qualité/prix), où ils gardaient de chauds partisans (la machine était " astucieuse " dans son principe alors que l'autre faisait plutôt appel à la force brute), et surtout dans la mesure où il n'y avait pas encore à faire de choix définitif ${ }^{17}$.

Les deux projets furent étudiés tout au long de 1962. En décembre de cette année, souhaitant que la décision définitive fût bientôt prise, la direction du CERN décida de formaliser le processus par lequel le choix serait fait. Pour diverses raisons (de prudence notamment), elle créa un Comité européen pour les futurs accélérateurs (ECFA), formé de représentants des communautés physiciennes de tous les États de l'Europe de l'Ouest. L'objectif confié à ce groupe d'une cinquantaine de personnes était de définir, du point de vue scientifique, le programme de construction nécessaire à la physique des hautes énergies européenne des années $1970^{18}$. Après six mois de travail, l'ECFA proposa une " pyramide " d'accélérateurs. Situé au sommet de celle-ci, le CERN se voyait doté de deux machines, un synchrotron de $300 \mathrm{GeV}$ et les ISR. La raison de ce choix (ou peut-être vaudrait-il mieux dire de ce "non-choix") était que les ISR seraient prêts quelques années avant le $300 \mathrm{GeV}$, qu'ils permettraient d'explorer la "fenêtre " des $50 \mathrm{GeV}$ dans le centre de masse - et que les physiciens ne se déclaraient pas hostiles aux ISR si ceux-ci ne mettaient pas en danger l'autre machine, plus importante. L'implicite de cette proposition était évidemment que l'argent pourrait être trouvé pour le programme d'ensemble. Le coût annuel de celui-ci était estimé à 1,6 milliard de francs suisses à l'horizon du milieu des années 1970 , dont 500 millions annuels pour un CERN doté des deux machines. Notons pour comparaison que le budget du CERN en 1963 était de 95 millions ${ }^{19}$.

16. Sur le ralliement des Européens, voir le très long compte rendu d'une réunion tenue au Royaume-Uni le 24/10/61, DSIR and NIRNS, Joint Consultative Panel for Nuclear Research (DG 20831).

17. K. JoHNSEN, Proposed Programme for a CERN Study Group on High Energy Projects, 27/11/61, Genève, CERN AR/Int. SR/61-29.

18. Lettre circulaire CERN/8187, envoyée le 30/11/62 par le directeur général à diverses personnalités européennes; Notes of Meeting of Sub-Committee for January Meeting on New Accelerator Programme, 30/11/62 (DG 20577).

19. Rapport du Groupe de travail sur le programme européen d'accélérateurs de haute énergie. Genève, CERN FA/WP/23/REV. 3, 12/6/63; ajouter ECFA, Notes for the Meeting, 9/10/64, FA/EC/6, p. 5 (DIRADM 20097). 
Cette confiance dans la capacité du CERN et de la communauté européenne des hautes énergies à obtenir de telles sommes ne tarda pas à apparaître quelque peu optimiste. Dès la fin de 1963, les gouvernements d'un certain nombre de pays manifestèrent leur intention de réduire leurs crédits scientifiques, notamment au Royaume-Uni; parallèlement, un nombre grandissant de spécialistes d'autres disciplines marquèrent leur désir de voir la part relative des hautes énergies ne plus croître. L'idylle post-Spoutnik touchait donc à sa fin. Une exception à noter dans cette évolution : la France gaullienne, alors prête à des projets nationaux ou européens (non américains) ambitieux ${ }^{20}$.

De juin 1963 au printemps 1964, le directoire du CERN (c'est-à-dire la direction du laboratoire) fit mine de ne pas comprendre ce retournement de conjoncture et continua à se faire l'avocat d'un programme "équilibré", formé de deux machines complémentaires, et dont aucune ne pouvait être abandonnée sous peine de catastrophe pour l'Europe. En mai 1964, toutefois, il se résolut à changer de « tactique de vente " en acceptant un échelonnement de ses demandes de financement. Son idée était d'obtenir un vote du Conseil des États en faveur des ISR immédiatement - dans la mesure où la somme demandée était moindre ( 300 millions contre un milliard et demi pour le 300 $\mathrm{GeV}$ ) ; puis de mobiliser toutes les forces - ce que nous avons caractérisé ailleurs comme le lobby-CERN - pour le $300 \mathrm{GeV}$. L'espoir était ici une décision à échéance de deux ou trois ans. En juin 1964, le directeur-général, Victor Weisskopf, demandait au Comité des directives scientifiques d'approuver cet échéancier ${ }^{21}$.

Notons avant de continuer notre récit que la direction du CERN entendait bien obtenir les deux machines et qu'il ne s'agissait pas pour elle de choisir l'une (les ISR) contre l'autre. Ce qu'elle souhaitait, et particulièrement le directeur général, c'était de sortir de la situation d'attente généralisée que le nouveau contexte avait créée, et de faire commencer effectivement les prises de décisions financières par les États.

Les physiciens européens et ceux du CERN réagirent très vivement à cette proposition de la direction. Dans leur quasi-unanimité, ils jugèrent le jeu beaucoup trop risqué. Les ISR accordés, dirent-ils en sub-

20. Howard Simons, "Britain Holds the Key to Expansion ", New Scientist, t. 393, 1964 , p. 542 ; pour la France, D. PESTRE, op. cit. supra, n. 4, p. 61-62.

21. Victor WEISSKOPF, Memorandum on CERN Accelerator Policy, 6/5/64, CERN/ SPC/183; sur la notion de lobby-CERN, D. PESTRE, "L'organisation européenne pour la recherche nucléaire (CERN) : un succès politique et scientifique? ", Vingtième Siècle, revue d'histoire, 4, 1984, p. 65-76; D. Pestre, J. KRIGE, op. cit. supra, n. 2. 
stance, les gouvernements pourraient se sentir quitte avec la physique des hautes énergies pour de nombreuses années. Or l'ECFA - et euxmêmes - avaient toujours été explicites sur un point : si les ISR étaient considérés, c'était à la condition expresse qu'ils ne missent jamais en danger une décision rapide à propos du $300 \mathrm{GeV}$. Puisque tel semblait être le cas, ils en demandaient le retrait pur et simple. En bref, et pour la première fois, un choix explicite était demandé22.

Les partisans des ISR et la direction du CERN réagirent en faisant valoir que cette logique de raisonnement était très pessimiste, que le $300 \mathrm{GeV}$ n'était pas " trop " urgent puisque la machine américaine de même type ne serait probablement décidée qu'en 1966 ou 1967 qu'en tous les cas un Tiens valait mieux que deux Tu l'auras. Au nom d'un certain réalisme, la direction obtint du Comité des directives scientifiques qu'il recommandât son projet au Conseil. Les 18 et 19 juin, ce dernier suivait l'avis de son comité scientifique. Il demandait aux gouvernements d'approuver le projet ISR, la décision sur l'autre machine étant momentanément repoussée ${ }^{23}$.

Les motivations des représentants non scientifiques des États au Conseil, lors du vote pour les seuls ISR, différèrent quelque peu de celles des scientifiques de la direction du CERN ou du Comité des directives scientifiques. Trois éléments les motivèrent particulièrement. D'abord les coûts : les ISR formaient un projet acceptable financièrement alors que l'autre était irréaliste ; ensuite, tous les paramètres politico-institutionnels étaient connus : les ISR seraient construits à Genève, dans le cadre du laboratoire existant; enfin, il y avait l'image qu'ils se faisaient des machines : comparés au $300 \mathrm{GeV}$, les ISR paraissaient extraordinaires. Ils étaient techniquement astucieux (on leur avait expliqué pourquoi depuis trois ans et demi), ils étaient originaux (puisque les Américains n'envisageaient pas d'en construire), et ils permettaient d'atteindre des énergies exceptionnelles. Que cela impliquât quelques contraintes (expérimentales, par exemple) n'était que contrepartie normale. En d'autres termes, ce fut pour des raisons qui leur étaient propres - du moins pour une pondération particulière de celles-ci - que les nouveaux venus dans la discussion soutinrent les anneaux d'intersection et tendirent à ignorer le $300 \mathrm{GeV}$. Percevant en partie la chose du point de vue de leur gouvernement, utilisant leurs propres critères de jugement et de rationalité -

22. Documents CERN/SPC/180/Draft, $8 / 4 / 64$, p. $4-5$; CERN/SPC/183, 6/5/64, p. 5 ; CERN/SPC/185/Draft, $1 / 6 / 64$, p. 8-9.

23. Document CERN/SPC/185/DRAFT, 1/6/64 ; Conseil des représentants des États, Procès-Verbaux des sessions du Conseil, séance des 18-19/6/64, Genève, CERN, 1965. 
à savoir rester compétitif pour aussi peu cher que possible - , ils définirent leur priorité - les ISR ${ }^{24}$.

Enclenché, le processus suivit alors son cours dans les divers appareils d'Etat. Certes, les physiciens ne se firent pas moins sévères dans leur critique mais ils durent toujours se soumettre dans leur désir de voir la décision renversée : pour les membres du Conseil, toute position "prématurée " et trop nette en faveur du $300 \mathrm{GeV}$ était maintenant inacceptable. Un changement de politique, au milieu du gué, aurait pour résultat de ruiner leur crédibilité dans les milieux gouvernementaux et de mettre à mal la confiance alors générale dans le CERN et son sérieux. L'exigence de réalisme politique imposait donc la poursuite du choix fait, imposait qu'on finançât d'abord le programme ISR.

Au Conseil de juin 1965, tous les États-membres, à l'exception de la Grèce, votaient ce programme de construction. Les physiciens acceptèrent alors, pour la première fois, de considérer sérieusement cette machine. Puisque 300 millions de francs étaient maintenant disponibles et que quelque chose du genre ISR devait être construit, ils étudièrent si ceux-ci pouvaient être notablement améliorés. Ils proposèrent plusieurs variantes durant l'été - minimisant notamment l'aspect collision. Elles furent toutes écartées par le Comité des directives scientifiques qui remarqua que l'étude technique sérieuse des nouvelles propositions nécessiterait un ou deux ans minimum, et qu'il était politiquement dangereux de ne pas commencer maintenant la construction permise par les 300 millions. En décembre, le Conseil confirmait cette position et la construction des ISR commençait ${ }^{25}$.

Pour résumer très sommairement la suite et la fin de l'histoire, notons que l'ECFA se réunit une nouvelle fois en 1966 et confirma le besoin impérieux d'un synchrotron à protons de $300 \mathrm{GeV}$. En 1967, 1'Autriche, la Belgique et la France annonçaient leur volonté de contribuer financièrement à sa construction. Pour sa part, la délicate question du site de cette machine de plusieurs kilomètres se déployait, de comité en comité, dans toute sa complexité. Au Conseil de juin 1968, les délégués britanniques informèrent leurs collègues que le gouvernement de Sa Majesté ne participerait pas au programme $300 \mathrm{GeV}$. Avant la fin de l'année, par contre, l'Allemagne, l'Italie et la Suisse

24. Conseil des représentants..., op. cit. supra, n. 23, p. 21-31.

25. Documents CERN/SPC/209/Draft, 23/11/65, p. 7-9; CERN/SPC/209/Draft/ Add., $13 / 12 / 65 ;$ CERN/SPC/217/Draft, $1 / 3 / 66$ (réunion tenue le 14/12/65), p. 5 ; Conseil des représentants des Etats, Pracès-verbaux des sessions du Conseil, séance des $15-16 / 12 / 65$, p. 55-57 et 57-60. 
s'étaient jointes à l'Autriche, la Belgique et la France : un projet à six - ouvert à d'autres - se précisait donc.

Début 1969, John Adams, le constructeur du PS de $25 \mathrm{GeV}$, accepta de devenir le directeur du programme $300 \mathrm{GeV}$. Il proposa un ensemble précis de procédures pour résoudre les diverses questions en suspens. Elles échouèrent au Conseil de décembre 1969, notamment sur le problème du site. La situation semblait définitivement bloquée au printemps 1970 .

Adams proposa une dernière donne, radicalement différente : installer le $300 \mathrm{GeV}$ à Genève - solution simple au problème du site -, et utiliser le PS du CERN comme injecteur - moyen très important de réduction des coûts. Le résultat en fut l'accord du Royaume-Uni non sans l'aide du secrétaire d'État « for Education and Science ", Margaret Thatcher. Les quatre grands étant alors dans la barque, il fut possible de « convaincre " les petits Etats non encore membres du projet (Pays-Bas, Suède). Le 19 février 1971, dix États-membres acceptaient le financement du nouvel accélérateur ${ }^{26}$.

Quatre années plus tôt, le financement d'un accélérateur de même type et de $400 \mathrm{GeV}$ avait été accepté par les autorités américaines. Sa construction avait commencé immédiatement. Le 1er mars 1972, il entrait en fonctionnement dans les plaines de l'Illinois, à une cinquantaine de kilomètres de Chicago. La machine européenne équivalente (qui fut finalement elle aussi de $400 \mathrm{GeV}$ ) ne fonctionna qu'en 1976 : elle avait quatre années de retard ${ }^{27}$.

\section{2. - ANALYSE DE LA DÉCISION ET COMMENTAIRES}

L'étude de notre cas particulier s'arrête ici. Ce que nous voudrions faire maintenant, c'est réfléchir plus globalement à ce qui est en jeu dans les décisions de gros équipements en "science lourde ". Nous porterons successivement notre attention sur quatre aspects. La nature de l'argumentation et des débats techniques d'abord, c'est-à-dire la manière dont les scientifiques abordent et concluent les questions internes à la discipline; ensuite l'articulation de ces considérations

\footnotetext{
26. Maurice Goldsmith, Edwin Shaw, Europe's Giant Accelerator, The Story of the CERN 400 GeV Proton Synchrotron, Londres, Tayler \& Francis, 1977.

27. James R. SANFORD, «The Fermi National Accelerator Laboratory ", Annual Review of Nuclear Science, t. 26, 1976, p. 151-198.
} 
avec celles qui sont tenues par les acteurs comme non techniques, les questions de coût par exemple, ou les développements qui ont lieu dans les laboratoires concurrents; puis la nature des corrélations entre les choix proposés et les places sociales occupées par ceux qui font ces propositions - exemple : comment rendre compte, et quel est l'effet, des variations d'attitudes apparaissant entre physiciens et constructeurs? ; enfin le processus en tant qu'il se déroule dans un temps long et acquiert, de ce fait, une inertie propre qui n'est pas réductible à la somme des espoirs ou des actes des participants.

\subsection{Les manières de penser techniquement les équipements lourds}

Ce dont les scientifiques débattent le plus, statistiquement, c'est de questions techniques. Leurs manières de penser, dans ce domaine, ne sont pas homogènes et deux grandes modalités sont identifiables. La première consiste à essayer de définir la machine à construire en fonction des questions centrales et non résolues de la discipline (celles que les physiciens se posent), en fonction de la question : "quel programme expérimental avons-nous à l'esprit ? " Illustrons ce que nous voulons dire à l'aide d'un exemple - et pour varier les plaisirs, prenons-le en dehors du champ des accélérateurs. En 1957, le CERN souhaitait déterminer les détecteurs de grande taille dont il aurait besoin dans les années 1960 pour faire de la physique avec son nouveau PS de $25 \mathrm{GeV}^{28}$. Après quelques réunions préliminaires, la manière dont il fallait aborder la question fut résumée par John Adams, alors directeur de la toute-puissante division construisant le PS.

«La manière correcte pour définir le programme de construction de l'appareillage expérimental, déclara-t-il en juillet, est d'abord de dresser une liste des expériences probables [à faire dans les années 60] et à partir d'une analyse de cette liste, de choisir l'appareil le plus utile, le plus adapté $" 29$.

28. Certains de ces équipements représentaient des entreprises coûteuses et techniquement sophistiquées : une chambre à bulles à hydrogène de 2 mètres par exemple - un détecteur qui fut construit au CERN entre 1959 et 1964 - pouvait contenir 6 ou 700 litres d'hydrogène liquide à $-246^{\circ} \mathrm{C}$, être munie de hublots de 15 centimètres d'épaisseur, être installée dans un électro-aimant de 200 tonnes produisant un champ de 12 kilogauss au centre de la chambre, etc. Pour une information plus générale sur les chambres à bulles, Luis W. Alvarez, « Recent Developments in Particle Physics ", in Marcello Conversi, ed., Evolution of Particle Physics, New York, Academic Press, 1970, p. 1-49. Pour un travail plus historique, Peter Galison, «Bubble Chambers and the Experimental Workplace ", in Owen Hannaway, Peter Achinstein, eds, Observation, Experiment and Hypothesis in Modern Physical Science, Cambridge, The MIT Press, 1985, p. 309-373.

29. J. B. ADAMS, rapport CERN/PS/DIR, 1/7/57, p. 4 (JBA 22798). 
Dans les mois suivants, cette procédure fut suivie et des listes furent établies qui servirent à comparer les mérites des divers types de détecteurs. Dans le cas des accélérateurs, on remarquera que c'est de cette manière que les physiciens abordèrent la question lors de leur conférence de juin 1961 (on en trouve confirmation dans les comptes rendus), et que c'est elle qu'on remarque à l'autre extrémité du processus, pendant l'été 1965 , lorsque les physiciens réouvrirent le débat presque clos à propos des ISR. Plus généralement, on la voit ressurgir régulièrement, notamment lorsque les scientifiques ont l'impression que le débat s'enlise.

Force est pourtant de constater que si les scientifiques ouvrent souvent leurs débats de cette façon, et y reviennent sans cesse, ceci ne les conduit que rarement à trouver une solution qui serait acceptée par tous, a fortiori bien sûr à trouver la solution qui serait définie du "strict point de vue scientifique ", comme ils aiment à dire. La raison en est que les appréciations et jugements qu'ils portent (sur le caractère typique ou non des expériences retenues pour comparaison, par exemple) souffrent d'un mal chronique, inévitable : l'ambiguitté voire l'arbitraire - qui découle de toute tentative d'extrapolation à huit ou dix ans à partir de théories et données expérimentales dont chacun s'accorde à dire qu'elles seront périmées au moment de la mise en service de la machine. Le résultat en est que ces approches n'autorisent, au mieux, que la création de consensus partiels sur des ordres de grandeur, ou la mise à l'écart de certaines alternatives. Un bon exemple de ceci est fourni par la conclusion que le directeur de la division Théorie du CERN, Léon Van Hove, tira de la conférence de juin 1961. $\mathrm{Ce}$ dont avaient besoin les physiciens, déclara-t-il, c'est d'un synchrotron à cible fixe et de haute intensité, la recherche des hautes énergies étant jugée comme moins urgente. D'où le refus des ISR et, dans le paradigme technologique d'avant l'été, un moindre intérêt pour un synchrotron de plusieurs centaines de $\mathrm{GeV}$ (dont l'intensité serait alors moyenne) que pour une machine de haute intensité et d'énergie de l'ordre de 50 à $100 \mathrm{GeV}^{30}$.

D'autres modalités de raisonnement tendent donc à exister en parallèle aux précédentes, à cohabiter avec elles. S'enracinant dans notre dernière remarque, elles visent à imaginer, non une expérimentation particulière, non quelle théorie tester, mais à considérer d'emblée des hypothèses générales sur les perspectives jugées les plus prometteuses

30. Que les physiciens soient très conscients des limites imposées par l'exercice d'extrapolation est par exemple clair in L. VAN Hove, op. cit. supra, n. 13, p. 422. 
et probables à moyen terme à l'intérieur du champ (ici la physique des particules). L'argumentation se mène alors essentiellement du " point de vue " des machines ou des types de machines, le but étant de trouver celle qui garantit le mieux contre l'imprévisibilité du futur. Dans le cas où une machine ou un type de machine apparaît comme assez " universelle ", c'est-à-dire comme assez souple, assez polyvalente dans ses possibilités d'usage, le problème se trouve largement simplifié. Dans le débat de 1957-1958, à propos des détecteurs à construire pour les synchrotrons de 25 et $33 \mathrm{GeV}$ du CERN et de Brookhaven, une argumentation de ce type fut avancée par Alvarez, un des grands hommes du laboratoire californien de Lawrence à Berkeley. Son point de vue était qu'aux énergies qui seraient disponibles avec la nouvelle génération d'accélérateurs, les détecteurs visuels (photographiant des traces d'événements) seraient supérieurs aux détecteurs dits électroniques, du fait même de la nature des phénomènes les plus intéressants qui seraient créés, du fait même du type d'événements dominants qui seraient produits. Face à eux, il essayait de montrer que les détecteurs électroniques seraient de plus en plus démunis ${ }^{31}$. Sa conclusion était qu'il fallait parier sur les chambres à bulles, de loin le meilleur détecteur visuel. "C'est [donc] une excellente approximation de dire, concluait-il, que dans le champ des hautes énergies, la chambre à bulles [deviendra] le détecteur universel ${ }^{32}$.

(Quelqu'un pourrait ici arguer - anticipant sur la suite de ce texte - qu'Alvarez était alors le chef de file des physiciens dont la tradition de recherche était dans les détecteurs visuels, et que son argumentation visait à justifier ses propres choix et à renforcer la force institutionnelle d'un des groupes en présence. Ceci est sensé mais n'évite pas notre propos : ce qu'Alvarez écrit est lu, repris, discuté, au CERN par exemple, et des opinions se font et se défont en fonction de ses écrits. La valeur intrinsèque de l'argument est donc considérée et c'est ce qui nous importe pour l'instant) ${ }^{33}$.

Dans le cas des accélérateurs qui nous a intéressé dans le récit, chacun se rappellera que c'est aussi cet aspect " universel " (dans le sens d'Alvarez) ou " multipurpose " (pour reprendre le mot des constructeurs) qui fit le succès du synchrotron de haute énergie auprès des physiciens à l'automne 1961. C'est, en effet, quand les constructeurs se

31. Pour une analyse un peu plus détaillée techniquement, cf. J. Krige, D. Pestre, art. cit. supra, n. 3, p. 258-259.

32. Lettre L.W. Alvarez à E. M. McMillan, 17/9/57, p. 18 (DG20551).

33. Ceci est particulièrement vrai au CERN où les opinions sont alors moins tranchées qu'aux Etats-Unis. Sur l'idée de deux traditions de recherches assez imperméables l'une à l'autre - détecteurs visuels et détecteurs électroniques - cf. P. Galison, op. cit. supra, n. 28. 
montrèrent convaincus, pendant l'été aux États-Unis, que ces machines seraient non seulement viables d'un point de vue technique, mais surtout qu'elles seraient intrinsèquement de haute intensité, qu'elles furent plébiscitées. L'intérêt pour les machines visant en propre l'intensité disparut alors. On pourrait ajouter, pour clore ce point, que plus le champ scientifique est ouvert et est traversé de programmes de recherche requérant des équipements différents, plus la tendance des scientifiques est de raisonner selon cette seconde modalité. Cela apparaît nettement dans l'étude sur la recherche astronomique des années 1945-1965 menée par Edge et Mulkay en 1976, et où traditions de recherche des divers groupes, choix stratégiques du champ à investir, et type d'appareillage sont très fortement corrélés ${ }^{34}$.

Tout ceci revient-il à dire que lorsqu'un type d'équipement est perçu comme "supérieur " aux autres, les questions de physique à faire avec lui perdent leur intérêt, que la première manière d'aborder les questions disparaît de la scène? Une telle conclusion semblerait hâtive - et largement fausse si l'on parle des physiciens. Il est, en effet, dans leur nature, pourrait-on dire, il en est de leur Être de chercheur de revenir toujours à ces questions d'expérience à faire, de théorie à tester. Imaginer ce qu'ils feront dans le futur, avec le nouvel accélérateur par exemple, est donc une préoccupation qui ne s'estompe jamais et reste en droit la première. On peut même dire que la question de la machine précise qu'ils souhaitent est, avec le temps, toujours plus liée au programme expérimental qu'ils envisagent pour elle. Donnons un bref exemple pour en comprendre la raison. En 1960, à propos des accélérateurs, les physiciens n'avaient de données sérieuses que pour des énergies oscillant entre 3 et $6 \mathrm{GeV}$ (la physique faite avec les machines du début des années 50). L'extrapolation à 100 ou $300 \mathrm{GeV}$ était donc particulièrement hasardeuse, et les physiciens se méfiaient de leurs propres prédictions. Vers 1964 ou 1965, par contre, la confiance en eux-mêmes était revenue - entre autres raisons, car ils avaient plusieurs années de travail à 25 et $30 \mathrm{GeV}$ et toute leur compréhension de la physique des particules en avait été modifiée. L'exercice d'extrapolation était donc vécu comme moins périlleux.

Une de nos premières conclusions est donc que diverses manières de traiter le sujet, diverses logiques de raisonnement (nous en avons vu nettement deux) sont à l'œuvre lorsque les scientifiques débattent techniquement d'équipements lourds à construire. Il y a rarement - voire jamais - une seule manière de poser le problème (même à l'échelle d'un individu) et qui serait linéaire et univoque pour réfléchir et abor-

34. D. O. Edge, M. J. MulkaY, op. cit. supra, n. 3, particulièrement p. 224-261. 
der la question. Ceci n'est toutefois pas propre aux questions de décision et caractérise au contraire tout effort de recherche. Le scientifique est toujours en position de devoir juger, de devoir choisir le modèle à utiliser, le groupe de données à privilégier, l'approximation la plus prometteuse. Souvent, il utilisera des approches différentes - et c'est seulement dans les mauvais livres et chez ceux qui considèrent Comte ou Berthelot comme ayant dit le Vrai de la Science et de sa Méthode que la recherche semble s'effectuer sur une voie royale, sans impasse ni détour, dans un monde où il n'est pas à estimer des potentialités, où il n'est jamais à prendre de raccourcis - où il n'y a pas aussi à parier sur des machines et des dispositifs expérimentaux.

Un dernier point avant de quitter ce niveau d'analyse. Dans le cas où un type de machines est reconnu très majoritairement comme la machine la plus " universelle ", le contenu de la décision se réduit souvent à décider d'un paramètre, la taille. Ceci a notamment été vrai pour les chambres à bulles dans les années 1950 et 1960 , et pour les synchrotrons à cible fixe de 1945 à nos jours. Dans ces cas, il est dit que plus la machine est grande, meilleure elle est du point de vue de la physique qu'elle permettra (la gamme des "événements" qu'elle autorise est a priori plus vaste). La fixation de la limite est alors largement faite en fonction d'autres types de considérations, techniques, économiques, psychologiques - ou purement fortuites ${ }^{35}$. Bien sûr, les scientifiques n'admettent pas toujours ce fait, en particulier dans les rapports qu'ils soumettent à l'extérieur. Le fait est pourtant indéniable et beaucoup le reconnaissent en privé ${ }^{36}$. Il conduisit, d'ailleurs, certains constructeurs à penser, dès 1961 et 1962, qu'il suffisait de multiplier par 10 l'énergie des synchrotrons à protons pour obtenir la règle de progression historique : 0,3 à $0,4 \mathrm{GeV}$ pour les synchrotrons des années d'immédiat après-guerre, 3 à $6 \mathrm{GeV}$ pour les synchrotrons mis en service entre 1952 et 1954,25 à $33 \mathrm{GeV}$ pour ceux opérationnels en 1959 et $1960, \ldots$ soit 3 ou 400 pour ceux qui étaient à décider. L'histoire, sérieuse, leur a peut-être donné raison ${ }^{37}$.

35. L'article de J. Krige et D. Pestre (op. cit. supra, n. 3) donne des exemples pour les chambres à bulles.

36. Le théoricien américain Robert SERBER déclarait en septembre 1961 que, lorsqu'on est dans ce cas, le choix de la taille « devient plutôt une question économique que de physique ». Il ajoutait : " nous préférerions aller aussi loin qu'il est techniquement possible sans demander quelque chose de trop déraisonnable au gouvernement ", International Conference on High Energy Accelerators, USAEC - BNL (?), 1961, p. 11 ; cf. aussi J. Krige, D. Pestre, op. cit. supra, n. 3, p. 268 (note 32).

37. Ceci est explicite dans M.G.N. HINE, "The CERN Proton Synchrotron, 1954-1962 : Forecasts and Reality Compared. An Informal Talk », in Rendiconti della Scuola Internazionale di Fisica "E. Fermi". New York, Academic Press, 1962, p. 287-294. Hine, directeur de la recherche appliquée au CERN, visait là directement les physiciens, incapables, selon lui, d’aider à la décision. 


\subsection{Les considérations jugées comme non techniques et apparaissant dans les débats}

D'autres considérations tout aussi décisives que les précédentes interviennent dans les jugements portés par les scientifiques lorsqu'ils débattent de gros équipements. Comme nous venons de le signaler, celles-ci prennent d'autant plus d'importance qu'on se trouve proche d'un cas où une machine est reconnue par la majorité comme la plus " universelle ». Il s'agit des capacités matérielles de réalisation (limitations techniques pouvant apparaître lors de la construction), des disponibilités en main-d'œuvre (négociables, s'entend, comme les précédentes, et jamais données définitivement), des coûts respectifs des diverses propositions (et de l'enveloppe finale attribuée ou escomptée), des temps de construction requis - l'ensemble étant marqué par l'état d'avancement des décisions et réalisations dans les centres concurrents. En bref, il s'agit de prendre en compte l'existence de ressources non illimitées qu'il faut utiliser au mieux dans le temps et l'espace afin d'être le premier dans la compétition.

Dans la physique des hautes énergies européenne des années 1950 et 1960 - et ici il semble qu'il faille être prudent avant de généraliser - ces considérations ne jouent pas les premiers rôles. Les estimations de coût, notamment, ne semblent pas occuper la place centrale qu'elles occupent dans le cadre de la radio-astronomie étudiée par Darmon et Lemaine. Considérant la décision d'un important interféromètre millimétrique dans les années 1960 et 1970, ils constatent que ces éléments de coût sont au cœur du processus de négociation dès les premiers instants. Formellement, les coûts sont censés être calculés après que le projet a été déterminé scientifiquement. De fait, ils sont largement « produits " en fonction de ce qu'il semble possible d'obtenir de l'État (en l'occurrence du CNRS). Dès le début, les considérations financières jouent donc comme une contrainte assez étroite ${ }^{38}$. Dans le cas des hautes énergies européennes des années 1950 et 1960, elles ne semblent, par contre, intervenir qu'à la marge, comme un groupe de conditions aux limites assez lointaines et vagues. Lors de la décision des chambres à bulles en 1958, par exemple, elles ne furent jamais discutées, la communauté tenant pour acquis que les sommes demandées seraient obtenues. À l'évidence, il faisait peu de doute que

38. G. Darmon, op. cit. supra, n. 3 ; G. Darmon, G. Lemaine, op. cit. supra, n. 3. 
le lobby des hautes énergies trouverait l'argent pour les deux chambres, dont celle de 2 mètres.

Dans le cas des accélérateurs de seconde génération, la même confiance dans la force institutionnelle du CERN domina la période jusqu'au début de 1964, nous l'avons vu. Certes, le récit a montré les effets " pervers " de cet optimisme dans les années suivantes. Mais le fait est là : jusqu'à cette date - et en particulier au nom du devoir pour le CERN de rattraper les Américains - le milieu n'a jamais mis au cœur de ses considérations les affaires d'argent. Un résultat indirect en est que, à la différence de ce qui a été souvent observé dans d'autres domaines scientifiques ou en recherche-développement, les physiciens des hautes énergies européennes ne semblent pas avoir en général minoré délibérément leurs estimations de coût. Convaincus de leur puissance, ils se firent un honneur d'être d'une grande fiabilité financière. On ajoutera que ceci ne fut possible que grâce à une forte auto-organisation de la communauté. C'est elle, organisée dans sa pyramide d'institutions, qui décidait au nom de l'intérêt supérieur du groupe. En d'autres termes, les francs-tireurs, comme l'appel direct d'un groupe particulier aux autorités financières, n'étaient pas admis. Et le système fonctionna de cette façon, assez bien.

L'argument du coût est pourtant utilisé dans le débat interne au milieu, évidemment par ceux qui se font l'avocat d'un projet meilleur marché. Ce que font les partisans des ISR, par exemple, est d'essayer de déplacer le cadre de la décision, de modifier les critères à l'aune desquels le jugement final devrait se faire. Ainsi argumentent-ils qu'il ne faut pas seulement demander : scientifiquement parlant " préféreriez-vous avoir des anneaux de stockage [ISR] ou un grand accélérateur [300 GeV]? ", mais, " en termes de rapport intérêt scientifiquecoût, les anneaux de stockage sont-ils un investissement raisonnable? " ${ }^{39}$. C'est pourquoi, à la limite, ils sont prêts à accepter la critique des physiciens qui veut que les ISR ne soient pas un accélérateur, mais une expérience proton-proton. Ils font, en effet, remarquer que leur coût est précisément de l'ordre de grandeur d'une série d'expériences avec un grand synchrotron - et que sous cet angle, les ISR sont remarquables.

La question de savoir quand les divers équipements proposés pourront être prêts est souvent plus importante car elle est étroitement imbriquée avec l'aspect hautement compétitif du travail scientifique. L'intérêt d'une chambre à bulles de 2 mètres par rapport à une de 1 mètre réside dans les potentialités expérimentales supérieures

39. Phrase de M.G. N. HiNE lors de la conférence citée, supra, n. 36, p. 287. 
offertes, nous l'avons dit. L'ennui est que les grands équipements sont souvent plus long à construire et que l'expérimentation avec eux en est retardée d'autant. D'où les craintes que d'autres soient prêts avant : « il ne sert à rien d'exceller, déclarait le directeur-général devant le Conseil du 13 juin 1962, si l'on excelle trop tard $"{ }^{40}$.

Le problème de choix qui résulte de ces exigences contradictoires est complexe dans la mesure où il n'est pas une seule manière de gagner dans la compétition scientifique. On peut notamment préférer les toutes prochaines batailles - ou " choisir " d'attendre en se préparant mieux. Lors de la décision des chambres à bulles du CERN en 1958, beaucoup de physiciens penchaient pour une chambre de l'ordre de 1 mètre, classique dans sa conception, mais pouvant être prête en même temps que le PS de $25 \mathrm{GeV}$. Cela était possible - c'est le choix que firent alors les Français en construisant, en un peu plus de deux ans et demi, une chambre de 81 centimètres dérivée d'une chambre américaine ${ }^{41}$ - et aurait permis de profiter de l'avance du CERN : le synchrotron de $33 \mathrm{GeV}$ de Brookhaven avait presque un an de retard sur son concurrent européen. D'autres, et notamment les constructeurs de la division en charge du PS, insistèrent sur les désavantages à moyen terme de ce choix. Dès 1963 ou 1964, tous les grands laboratoires concurrents auraient des chambres de 2 mètres - et l'avantage momentané se transformerait en désavantage durable. Leur préférence allait donc à un sous-équipement initial (pas de chambre-CERN entre 1960 et 1963 ou 1964), largement compensé par la suite ${ }^{42}$.

Le même type de question apparut avec les séparateurs nécessaires à l'obtention de faisceaux secondaires de qualité. Le choix du CERN fut de construire des séparateurs (électrostatiques) sophistiqués, polyvalents, plus prometteurs sur le long terme - mais plus longs à construire et critiqués par d'autres comme "overdesigned". Au Brookhaven National Laboratory, le choix fut symétrique : séparateurs plus simples, moins souples, mais qui furent prêts plus tôt ${ }^{43}$. Ajoutons que la pertinence de cette exigence - "être prêt à temps " - varie en fonction inverse de l'importance intrinsèque de l'équipement. Six mois ou un an de différence pour une série de séparateurs tendent à être considérés par les expérimentateurs comme plus dommageables que deux ans pour un accélérateur d'un milliard de francs

40. Conseil des représentants des Etats, Procès-Verbaux des sessions du Conseil, séance du 13/6/62, Genève, CERN, 1963, p. 17.

41. "Numéro spécial consacré aux chambres à bulles françaises au CERN », L'onde électrique, t. 41 , déc. 1961.

42. J. KRIGE, D. Pestre, op. cit. supra, n. 3, p. 265-274.

43. Travaux à venir de J. KRIGE. 
suisses et dont la construction durera environ huit ans. Dans le premier cas, les avantages techniques supplémentaires ne sont pas souvent considérés par les physiciens comme "valant" un long délai d'utilisation. Dans le second, ils acceptent plus facilement l'idée de commencer à expérimenter à $300 \mathrm{GeV}$ après huit ans de construction, au lieu de travailler à $150 \mathrm{GeV}$ après six ans ${ }^{44}$.

Ceci conduit à raffiner quelque peu cette notion de compétition. Dans l'abstrait, chacun " joue " contre chacun. En réalité - et si on nous permet ici la métaphore du sport - , il y a des hiérarchies et certains laboratoires sont en "première division". Certes, comme dans un match de coupe, des équipes de divisions différentes peuvent être amenées à se trouver en compétition autour d'un même type de phénomène ou de question. En pratique toutefois, cela est rare. Dans les années 1950-1965, le CERN est dans le cas du laboratoire qui vient d'être promu dans un groupe de première division exclusivement américain, et qui souhaite démontrer qu'il ne l'a pas été par hasard. Une inquiétude, une certaine préoccupation existe donc; en fait, on pourrait presque parler d'obsession vis-à-vis de tout ce qui se passe dans les grands laboratoires d'outre-Atlantique. Ce sentiment conduit à ce que soient moins prises en compte, lorsqu'il s'agit de décider d'un équipement de base devant être opérationnel sur une longue période, les demandes de ceux qui privilégient les solutions immédiates. Dans le cas des chambres à bulles par exemple, le groupe d'Alvarez était dominant : en 1959, il disposait déjà d'une chambre à hydrogène de 72 inches (environ $1,80 \mathrm{~m}$ ). Devoir devenir compétitif "impliquait" donc pour le $C E R N$ (mais cela était moins vrai pour la France, par exemple) de privilégier le long terme et de ne pas considérer une chambre inférieure en taille à celle de Berkeley. Certes, un ou deux ans pouvaient être gagnés avec une chambre moins ambitieuse mais le retard vis-à-vis du meilleur ne serait jamais que partiellement comblé.

Une logique identique prévalut en 1950-1953 lorsque fut créé le CERN et décidé le PS de $25 \mathrm{GeV}$. En décembre 1950, la première proposition fut explicitement de faire au moins aussi bien que le laboratoire mondial le plus avancé, celui de Lawrence à Berkeley ${ }^{45}$. Un chiffre de 6 à $10 \mathrm{GeV}$ fut donc retenu - ce qui conduisit à une estimation de 60 millions de francs suisses. L'argent fut définitivement voté par

44. Cf. C. J. Zilverschoon, Draft Cost Estimate for a $300 \mathrm{GeV}$ Proton Synchrotron, Genève, CERN AR/Int. SG/63-4, 6/2/63 (respectivement $150 \mathrm{GeV}$, Ar/Int. SG/63-5, 6/2/63).

45. D. Pestre, "The Fusion of the Initiatives, June-December 1950 ", History of CER N, op. cit. supra, n. 2, p. 97-122. 
les Etats au Conseil de juin 1952. L'histoire continue pourtant. Deux mois plus tard, un élément technique nouveau apparaissait : une autre possibilité de focalisation des faisceaux. L'effet direct en était une réduction de la section de la chambre à vide, donc de la taille de la machine (champ magnétique plus grand), et donc de son prix! Le groupe technique en charge de la construction du PS au CERN décida d'adopter immédiatement le nouveau principe. Il considéra, toutefois, que les 60 millions étaient acquis et fit donc les plans de la machine la plus grande possible et valant cette somme. Il arriva ainsi au chiffre de $25 \mathrm{GeV}^{46}$.

Ce dernier cas présente en commun avec celui des chambres à bulles en 1958 d'être fortement marqué, voire déterminé par l'équipement dont dispose (ou va disposer) le laboratoire concurrent le plus puissant, en l'occurrence toujours américain. Dans le cas des accélérateurs de la seconde génération au CERN, par contre, il n'y a pas de point fixe à atteindre et les décisions européennes et américaines opèrent simultanément : tout mouvement d'un côté de l'Atlantique a quelque effet sur les projets de l'autre côté. Il reste, néanmoins, une certaine asymétrie, psychologique pourrait-on dire : l'inquiétude dont nous parlions précédemment ${ }^{47}$. Celle-ci ne devait, en fait, disparaitre que très lentement $:$ il fallut jusqu'au début des années 80 pour que les physiciens européens perdent totalement ce sentiment.

\subsection{Qui dit quoi? choix, positionnement social, intérêt}

Le récit a montré qu'il était impossible de rester dans le simple monde du discours, dans le monde des arguments échangés et invoqués. Certes, le royaume des idées a sa cohérence et sa logique qui interdisent qu'on puisse jamais en rendre compte socialement de façon complète. Il n'empêche : des corrélations apparaissent nettement quand on compare attitude et positionnement social, professionnel, institutionnel, national, etc. Ceci n'est, à vrai dire, guère surprenant si on ne considère pas les scientifiques comme de purs esprits animés d'une rationalité unique ou monomaniaque. Schématiquement, ce que font les scientifiques, c'est de fabriquer, de préciser, de contester des modèles qui rendent compte de régularités ou de variations obtenues à travers des dispositifs expérimentaux conçus par eux.

46. J. KRIGE, "Case Studies of Some Important Decisions ", History of CERN, op. cit. supra, n. 2, p. 237-292, ici p. 274-276.

47. Ne donnons ici qu'une référence d'archive relative à l'attitude d'Edoardo Amaldi, un des fondateurs du CERN, lors de la réunion du Comité des directives scientifiques du 10 octobre 1963. Cf. CERN/SPC/178/Draft, 17/1/64, p. 11. 
Dans ce travail, ce qui est élaboré est tributaire de règles formelles, mais aussi d'exigences heuristiques, de traditions particulières qui varient suivant les spécialités pratiquées (physique mathématique, physique théorique pure ou plus phénoménologique, physique expérimentale de type "électronique " ou de type "chambre à bulles", construction d'accélérateurs, conception de nouveaux détecteurs, ...), suivant les écoles, suivant les moments, suivant les générations, suivant les institutions d'accueil, etc. Les manières d'aborder les questions ouvertes du futur, de les formuler, de les résoudre, ont donc toutes les chances d'être marquées par ces positions et les traditions qui y conduisent.

\section{Corrélations entre attitudes et places occupées}

La corrélation la plus nette et la plus souvent notée par les acteurs eux-mêmes lors du débat sur les accélérateurs, met en rapport prises de positions et trois grandes spécialités, trois grandes formations de base : physiciens expérimentateurs, physiciens théoriciens, ingénieursconstructeurs. Cette première subdivision est nettement simplificatrice, étant trop lâche - il existe différents types de théoriciens - et trop stricte - tous les spécialistes des machines ne font pas la même analyse. Elle donne pourtant la clé la plus permanente et la plus discriminante pour notre propos. Dans les années 1950 et 1960 , c'est le clivage entre physiciens (c'est-à-dire ici théoriciens et expérimentalistes) et constructeurs qui domine, nous l'avons établi ; par la suite, c'est celui qui sépare les expérimentateurs des théoriciens et des constructeurs. Ce dernier résultat, empirique, est celui auquel sont arrivés Jurdant et Olff-Nathan à propos de la décision du LEP (l'accélérateur actuellement en construction au CERN) à la fin des années 1970. Ce "glissement " de l'essentiel des théoriciens entre la seconde moitié des années 1960 et le début des années 1970 n'est pas surprenant. Il reflète assez directement le changement de statut qu'a connu la physique théorique des hautes énergies durant cette période. Devenue beaucoup plus puissante et synthétique (par opposition à classificatoire), elle a produit des prédictions très précises demandant, pour être vérifiées, des situations expérimentales extrêmes. Ceci a permis aux théoriciens et aux constructeurs de se trouver souvent derrière les mêmes projets face aux expérimentalistes ${ }^{48}$.

48. Baudoin JURDANT, Josiane OlfF-Nathan, Le LEP entre la physique et les physiciens, Strasbourg, GERSULP, texte ronéotypé, 1981 ; à propos de l'évolution du statut de la physique théorique, Andrew PICKERING, Constructing Quarks, Edinburgh, Edinburgh University Press, 1984. 
Il ne faudrait pourtant pas considérer que ces différences ont un caractère d'absolu. Elles semblent, par exemple, beaucoup moins marquées aux États-Unis qu'en Europe. Ici, on peut dire qu'un fossé existe entre constructeurs et physiciens; là, si différence il y a, elle est moindre, moins institutionnalisée, moins ancrée dans les pratiques et mentalités. Cela renvoie, en première approximation, à deux choses. D'une part, à des manières différentes, intellectuelles ou culturel!es pourrait-on dire, d'aborder les questions. Nous rangerions sous cette rubrique les approches décrites souvent comme plus pragmatiques des Américains - et dont une étude récente de Schweber a mis en évidence de très belle façon les effets sur la pratique théorique des physiciens entre 1930 et $1950^{49}$. De l'autre, à l'expérience de guerre et à ses demandes finalisées, ses dépenses massives, sa nécessité de combiner toutes les approches pour réussir vite, avant les nazis. Le résultat en fut, dans les années 1950 et 1960, une tendance constante de nombreux physiciens américains à considérer le développement de l'appareillage et des technologies comme partie intégrante de leurs activités, et donc à ne pas laisser aux "constructeurs " le champ que leurs collègues européens leur laissaient ${ }^{50}$.

Les effets de cette différence sont d'ailleurs perceptibles. Les enquêtes menées par Irvine et Martin ont par exemple montré que la très grande majorité des scientifiques (américains comme européens) considéraient les matériels lourds construits au CERN comme plus fiables, plus raffinés d'un point de vue technologique, plus susceptibles d'adaptation car conçus par des spécialistes - le revers en étant souvent leurs coûts et leurs temps de construction supérieurs ${ }^{51}$.

Ces clivages d'attitudes entre expérimentateurs, théoriciens et constructeurs - et Européens-Américains - ne sont pas les seuls. Le récit a suggéré des attitudes variables suivant l'âge ou les positions institutionnelles. Dans l'ensemble, par exemple, les physiciens du Comité des directives scientifiques ont toujours montré une position moins catégorique que celle des physiciens du CERN ou des États-membres,

49. Sam. S. Schweber, « The Empiricist Temper Regnant : Theoretical Physics in the United States, 1920-1950 », Historical Studies in the Physical and Biological Sciences, 2, 1987.

50. Devant l'ampleur de la bibliographie, nous renvoyons seulement à Daniel J. KEVLES, The Physicists, New York, A.A. Knopf, 1978. Pour des approches autobiographiques confirmant ces points, L.W. Alvarez, op. cit. supra, n. 28 ; Robert R. WiLson, " My Fight Against Team Research "; Gérard HoLton, ed., The Twentieth Century Physics, New York, W.W. Norton, 1972, p. 468-479.

51. Ben R. MARTIN, John IRvine, « CERN : Past Performance and Future Prospects I. CERN's Position in World High-Energy Physics », Research Policy, t. 13, 1984, p. 183-210 ; « id. - II. The Scientific Performance of the CERN Accelerators », Research Policy, t. 13, 1984, p. 247-284. 
ils furent plus enclins à chercher des solutions de compromis, à admettre l'argument du "réalisme " - en bref à jouer leur fonction de direction! L'évolution de l'attitude de Bernard Gregory serait ici intéressante. Jeune physicien, spécialiste des chambres à bulles, il fut le président du Comité des expériences avec chambres à traces du CERN en 1961, membre du directoire du CERN deux ans et demi plus tard et nommé directeur-général en 1965. Toujours en très grande sympathie avec les physiciens dans le débat ISR $/ 300 \mathrm{GeV}$, il fut l'un de ceux qui prêcha le réalisme (la résignation serait un peu fort) et contribua à l'acceptation des anneaux de stockage par ses collègues ${ }^{52}$.

Ces corrélations étant notées, comment en rendre compte (de façon plus générale que dans le seul cas de Gregory)? Dans certaines situations, on peut prétendre que ce sont des intérêts bien compris (au sens d'avoir intérêt à) qui contribuent à voir certains groupes ou certaines institutions adopter de préférence certaines positions. Par exemple, un des arguments que la direction du CERN fit régulièrement valoir entre 1957 et 1960 (toutes les fois qu'il s'est agi d'accepter ou de refuser la mise en route d'un projet d'envergure) fut que ces projets permettraient d'offrir un travail intéressant aux constructeurs qu'elle avait rassemblés pour construire le PS de $25 \mathrm{GeV}$. Ceux-ci constituaient un " pool " d'experts unique en Europe qu'elle ne souhaitait pas voir dispersé. Garder ce groupe à demeure et éviter son émigration aux États-Unis était donc un argument en soi et il apparut en tant que tel dans le débat à propos des chambres à bulles (en faveur d'une chambre de 2 mètres), dans le débat à propos du Groupe de recherche sur les accélérateurs (pour sa création) et dans celui à propos du groupe de travail sur la physique des plasmas (pour l'extension de ses activités) ${ }^{53}$.

Il est pourtant des raisons plus essentielles à certaines des corrélations constatées, et notamment à celle qui place en 1961, constructeurs et projet ISR d'une part, physiciens et synchrotron de l'autre. Pour les saisir, demandons-nous d'abord pourquoi les physiciens montrèrent un faible intérêt initial (fin 1960-début 1961) pour la question des accélérateurs de seconde génération. La réponse est triviale : ils commençaient alors à faire de la physique avec le tout nouveau PS de $25 \mathrm{GeV}$ (c'est l'époque où se précisait notamment la grande expérience neutrino) et la prochaine machine n'était pas leur affaire. Était-ce une attitude à courte vue comme certains constructeurs vou-

52. D. Pestre, op. cit. supra, n. 4, p. 84-87, 91-93.

53. ID., "Un autre aspect de la dimension européenne du CERN, "the European Study Group on Fusion ", 1958-1964». Etude annexe à D. PESTRE, op. cit. supra, n. 4, p. 96-104. 
laient le faire croire? Nous ne le pensons pas : elle était dans la logique des choses, celle de physiciens impatients d'aborder leur « frontière ". Jusqu'à présent, en effet, ils n'avaient travaillé que dans la gamme 3-6 GeV. Comment imaginer, dans ces conditions, qu'ils pussent déjà s'intéresser à des énergies de $300 \mathrm{GeV}$ et considérer la génération $x+2$ de machines - alors que l'accélérateur de génération $x+1$ était tout juste terminé et qu'ils ne connaissaient que la physique de génération $x$ ! "Logiquement », pourrait-on dire, au regard de ce qu'était le cœur de leur travail, c'est utiliser le nouveau PS qu'ils désiraient, et non concevoir ce qu'ils feraient en 1970 ou en 1980. Les machines de l'ordre de $30 \mathrm{GeV}$ étant terminées, les constructeurs regardaient, par contre, dans l'autre direction : ce qui n'était qu'un but pour les physiciens (utiliser ces machines de $30 \mathrm{GeV}$ ) était pour eux quelque chose de maitrisé puisque la machine fonctionnait, quelque chose de fini à partir duquel on devait aller de l'avant. À leurs yeux, il n'y avait pas à attendre et il fallait débattre, maintenant, de la machine suivante. Ne fallait-il pas, d'ailleurs, huit ou dix ans pour la construire ? On voit donc - et c'est ce que nous souhaiterions souligner - que la différence d'attitude entre physiciens et constructeurs se comprend en partie en référence à des positions particulières par rapport au savoir : quand une nouvelle machine entre en service, il y a comme "deux générations de machines d'écart " entre les uns et les autres.

Les différences entre les deux groupes ne se réduisent pourtant pas à cet écart-là. Ṃ̂ême lorsqu'ils considèrent le même problème - ici la machine de génération $\mathrm{x}+2$ - leurs préoccupations spontanées les conduisent, les tirent dans des directions non nécessairement identiques. Ce qui motive, ce qui préoccupe, ce pourquoi on accepte de passer des nuits blanches, diffère. Pour les uns, c'est d'abord la machine, pour les autres, c'est l'usage de cette machine; pour les uns, c'est d'abord la technologie de la machine, sa conception, pour les autres, la technologie des expériences qu'elle permettra ou interdira ; pour les uns, c'est plus l'objet en tant qu'objet, pour les autres, les potentialités pour jouer avec cet objet. Certes, chacun est sensible à la logique de l'autre : la subtilité du projet ISR séduit les physiciens que sont Perrin et Amaldi, et les exigences de flexibilité et d'intensité convainquent Adams en juillet 1961. Mais il y a des réflexes, des fascinations, des intérêts - dans le double sens de s'intéresser à quelque chose et d'y avoir intérêt cette fois - et les deux groupes tendent à s'éloigner. 


\section{Stratégies de groupes}

Dans certains cas, il est nécessaire d'aller plus loin que simplement constater des corrélations entre positionnement social et convictions intellectuelles, et de noter que des groupes socio-professionnels ou des institutions développent des stratégies pour convaincre d'autres groupes, ou imposer directement leurs choix. Donnons deux exemples.

En juillet 1961, la majorité des constructeurs dut reconnaître que sa proposition de décider rapidement des ISR était rejetée par tous les physiciens européens s'étant exprimés. Cela ne la conduisit pourtant pas à abandonner son idée. Convaincue de sa richesse potentielle et stimulée par l'intérêt qu'un physicien américain de renom, G. K. O'Neil portait à ces ISR - elle s'organisa pour les maintenir au programme. Dès l'automne 1961, elle préparait un séminaire des " enthousiastes des collisions de faisceaux", séminaire de quinze jours qui se tint au CERN en mars et avril 1962 en présence de $\mathrm{O}^{\prime} \mathrm{Neil}^{54}$. L'objectif de la réunion était de considérer la machine du point de vue de son utilisation, et donc de montrer aux physiciens sceptiques en quoi l'expérimentation était techniquement possible, en quoi la qualité des données serait suffisante, en quoi la complexité de celles-ci ne serait pas d'un ordre de grandeur supérieure à celles généralement obtenues. En d'autres termes, il s'agissait pour les constructeurs de convaincre les physiciens "sur leur terrain ", de leur prouver qu'il était intéressant pour eux - c'est-à-dire pour leur compréhension de certaines questions de physique comme pour leur carrière de faire de la physique avec les ISR.

Le second exemple a trait à la décision des chambres à bulles et permet d'illustrer une stratégie d'institution dont le but est cette fois moins de convaincre intellectuellement que d'imposer ses propres choix. Lors de la définition de son programme en 1958, le CERN tint à affirmer son identité naissante et à exister pleinement face à ses concurrents américains. Il réagit donc assez froidement à ce qui se passait dans les laboratoires des États-membres. En avril 1958, le directeur du laboratoire de l'École polytechnique de Paris, Louis Leprince-Ringuet, informa le directeur général du CERN qu'il souhai-

54. Experimental Use of Proton Storage Rings, Report of a Working Party Meeting from March 26 to April 6, 1962, Genève, CERN AR/Int. SG 62-11, 3/9/62; le mot d' " enthousiastes des collisions de faisceaux " est de K. JOHNSEN, constructeur des ISR, dans CERN/SPC/157/Draft, S/4/62, p. 12. 
tait amener auprès du PS de $25 \mathrm{GeV}$ une chambre à propane de 1 mètre. Pendant douze mois, la proposition fut «ignorée ", le CERN décidant de construire indépendamment une chambre de même type. De même, une suggestion faite par les Britanniques que le CERN collaborât avec eux à la réalisation de leur chambre à hydrogène de 1,5 mètre - le but étant d'augmenter les chances d'avoir une grande chambre à hydrogène prête à temps en Europe - fut repoussée. En l'occurrence, le CERN préféra construire sa propre chambre de 2 mètres. Le résultat de ces volontés institutionnelles privilégiant chacune leur programme (le CERN n'est guère plus en cause que les deux autres groupes) fut, dans les années suivantes, la multiplication des débats de priorité autour du thème : qui pourrait expérimenter autour du PS, quand, avec quelle chambre et à quelles conditions ${ }^{55}$.

Pour conclure sur ces analyses, nous aimerions souligner un point méthodologique essentiel à nos yeux, à savoir qu'il n'y a pas à tenter de rabattre systématiquement les divers éléments les uns sur les autres. Si nous avons distingué plusieurs niveaux dans l'étude, c'est que nous pensons qu'ils disposent d'une réelle autonomie. Ainsi, vouloir ignorer l'argumentation proprement technique au profit exclusif de considérations financières ou du désir de rester compétitif, vouloir réduire totalement la logique propre de la démarche intellectuelle à des jeux de pouvoir ou des stratégies de groupes, ou inversement, ne traiter la dimension pleinement sociale, institutionnelle ou «tactique " de la décision que sur le mode anecdotique - ce qui permet de considérer qu'elle ne touche pas à l'essence du résultat - nous semblerait également se condamner à ne pas comprendre. Il est plus riche, heuristiquement, d'accepter que la production des savoirs soit décrite à la fois comme " une authentique construction sociale qui puisse pourtant être une représentation du monde naturel sur laquelle on puisse compter $"{ }^{56}$. Peut-être apparaît-il difficile d'articuler théoriquement ces diverses dimensions dans un modèle pleinement satisfaisant pour l'esprit de géométrie ; cela ne peut pourtant pas autoriser à mettre, comme le dirait Amiel, "l'école [ou] la formule [...] au-dessus du réel et préfère[r] la clarté à la vérité [ou] les mots aux choses [...] " ${ }^{57}$.

55. D. PESTRE, Organiser l'expérimentation autour du synchrotron à protons, 1960-1965. La période d'apprentissage, Genève, CERN-CHS-22, 1987, p. 9-30.

56. Martin J. Rudwick, The Great Devonian Controversy, Chicago, Chicago University Press, 1985, p. 455.

57. Amiel, cité par Théodore ZELDIN, Histoire des passions françaises, Paris, Seuil, 1978 , t. 2, p. 233. 


\subsection{L'analyse en termes de processus}

On pourrait être tenté de résumer ou de visualiser l'analyse que nous avons présentée jusqu'à maintenant à partir du modèle du parallélogramme des forces de la statique. La manière d'expliquer la décision consisterait alors à dresser une liste des divers acteurs en présence, des diverses forces à l'œuvre ; à évaluer leur importance respective, ou leur poids relatif; à montrer le but poursuivi par chacun d'eux, ou l'objectif visé - la composition de ces divers vecteurs-forces produisant une résultante : le choix final. Une telle démarche présenterait malheureusement une faiblesse radicale en ceci qu'elle produirait une image, une reconstruction des événements qui serait " trop" rationnelle et nécessaire, qui ignorerait la dimension temporelle, la dimension de processus qu'a toujours une décision de ce type : de fait, il ne s'agit jamais de décider, tous ensemble, à un moment donné, de tout le problème. Si donc on souhaite parler en terme de résultante, il faut aussi la concevoir comme le dernier produit d'une succession de décisions intermédiaires semblant n'impliquer chacune que des conséquences limitées, comme le dernier élément d'une chaîne de choix locaux étalés dans le temps et ne répondant souvent qu'à des contextes "particuliers". En d'autres termes, le système de négociation que forment les acteurs doit aussi être perçu comme ayant une dynamique propre (chaque moment tend par exemple à contraindre toujours plus le suivant, à réduire les libertés de choix à venir), dynamique qu'on ne peut identifier à celle que tentent d'imposer les acteurs, dynamique que le modèle implicite de l'équilibre des forces ignore $^{58}$.

La raison pour laquelle cette dimension de processus est souvent ignorée, est que l'image spontanée qu'on se fait d'une décision rationnelle - et la décision d'un équipement par des scientifiques n'en forme-t-elle pas le meilleur prototype? - dérive de la notion de choix libre chère à la pensée occidentale depuis au moins le XvIIe siècle. Procédant de façon linéaire, logique, l'homme raisonnable définit d'abord

58. Sur l'analyse des décisions en termes de processus, cf. Graham T. Allison, Essence of Decision : Explaining the Cuban Missile Crisis, Boston, Little, Brown \& Co, 1971; Robert F. Coulam, Illusions of Choice : the F-111 and the Problem of Weapons Acquisition Reform, Princeton, Princeton University Press, 1977; Michel Crozier, Erhard Friedberg, L'Acteur et le Système, Paris, Seuil, 1977 ; G. Darmon, G. Lemaine, op. cit. supra, n. 3; Charles E. Lindblom, "The Science of “Muddling Through" ", Public Administration Review, t. 19, 1959, p. 79-88; Warner R. ScHILLING, « The H-Bomb Decision. How to Decide Without Actually Choosing ", Political Science Quarterly, t. 76, 1961 , p. $24-46$. 
des objectifs; en fonction de ses fins, il envisage les moyens disponibles et les compare; en résulte le choix optimal qu'il exécute. À chacune des étapes, en droit séparables même si elles s'enchaînent nécessairement, il est censé s'informer pleinement (présupposition d'information totale), être conscient des effets qu'auraient ses actions (présupposition de transparence des actes) et être logique et cohérent dans sa décision finale. En bref, il fait un choix optimal-rationnel dans une liste exhaustive de possibles bien définis ${ }^{59}$.

L'étude de cas que nous avons présentée montre bien les limites de ce type d'hypothèses. En effet :

1 - Les choix successifs sont faits par les acteurs sur la base d'alternatives souvent implicites, limitées en nombre, et dont la qualité est inégale. Rappelons que la proposition ISR de décembre 1960 résulte d'un groupe de scientifiques qui ne sépare pas fins et moyens (il y a nécessité d'un nouvel accélérateur/celui-ci doit être des ISR). La réaction des physiciens est de refus car il n'y a pas urgence et qu'une autre possibilité n'a pas été considérée, le synchrotron de haute intensité. Après l'été toutefois, cette alternative est dépassée car un autre type de machine est apparu, une machine plus polyvalente, le synchrotron de haute énergie. On le voit, l'information dont disposent les acteurs n'est jamais ni complète, ni stable, la liste des "moyens " n'est jamais close - au contraire ce sont presque toujours des propositions particulières ou des décisions provisoires qui suscitent et font naître de nouvelles solutions politiques ou techniques. Confirmation éloquente de ce fait serait donnée par l'attitude des physiciens durant l'été 1965 : c'est seulement lorsque la décision définitive de construire les ISR fut prise par les États qu'ils se penchèrent sérieusement sur le projet lui-même et proposèrent ... de le remplacer par d'autres jamais considérés jusque-là.

2 - Ces attitudes tiennent en partie au fait que les acteurs s'investissent très différemment dans le débat. Le temps est une denrée rare, chacun le sait, et les acteurs n'accordent pas la même attention aux mêmes questions au même moment. Les constructeurs, par exemple, pensent beaucoup plus aux accélérateurs de seconde génération que les physiciens, nous avons vu pourquoi. Que ces derniers aient donc souvent une information moindre que les premiers, qu'ils suivent le déroulement du processus de décision de façon plus épisodique et lointaine, qu'ils agissent donc surtout en réaction aux propositions des

59. Lucien Sfez, Critique de la décision, Paris, Presses de la Fondation nationale des sciences politiques, 1981 . 
constructeurs ou aux décisions intermédiaires n'est pas pour surprendre. Et s'il leur arrive d'intervenir " trop tard " (comme en 1965), c'est largement dans la logique des choses.

3 - Le récit a aussi montré qu'il n'y a pas une manière de poser le problème, mais plusieurs, que les fins, les enjeux et les critères considérés comme décisifs varient avec les individus et le temps. Il ne peut donc y avoir une rationalité de la décision qui soit à l'œuvre du début à la fin, mais des rationalités, souvent aussi respectables les unes que les autres, et qui s'opposent ou se succèdent. Un résultat en est que ceux en position de devoir " trancher " tendent à la prudence, tendent à procéder par étapes et à obtenir des consensus partiels qui heurtent le moins possible les jugements des groupes concurrents. Juxtaposer les projets et repousser à des jours meilleurs le moment où il faudra "vraiment choisir" est alors une démarche courante. Comme l'a fait remarquer Schilling, on est alors souvent conduit à savoir « comment on décide sans jamais vraiment choisir $"{ }^{60}$.

4 - Ce ne sont donc jamais les mêmes critères de choix, les mêmes déterminations, les mêmes groupes qui sont décisifs. C'est le moment auquel on se trouve dans le processus qui fait apparaitre les enjeux réels et donc définit les acteurs et les argumentations qui auront le plus de poids. Ceci peut, bien sûr, conduire à des résultantes en partie aléatoires ou à ce qu'on a coutume d'appeler des "effets pervers". Reprenons sur ce point le tournant de 1964. Au début du printemps, la crainte de ne rien obtenir du tout conduit le directoire du CERN à considérer un possible échelonnement dans le temps des projets ISR et $300 \mathrm{GeV}$. En mai, quoique hésitant, le directeur général soumet une proposition en ce sens. En juin, le SPC la fait sienne et la transmet au Conseil qui l'approuve quelques jours plus tard. Pendant l'été, les machineries étatiques européennes se mettent en branle pour dégager les crédits nécessaires aux ISR. À l'automne, les physiciens réagissent vivement - pour constater leur impuissance à inverser le cours des choses : on s'oriente dans chaque Etat vers un financement des ISR, mais contre tout projet de type $300 \mathrm{GeV}$ ! Un enchaînement de microdécisions a donc produit une macro-résultante qui, aux yeux de ceux désignés depuis le début comme devant, en droit, prendre la décision finale, va à l'encontre des souhaits qu'ils ont toujours exprimés. Au regard de la logique de décision que les acteurs avaient prétendu vouloir suivre (le choix final relève des seuls utilisateurs, les physiciens),

60. W.R. SChilling, op. cit. supra, n. 58 . On notera que ceci forme une conclusion essentielle à laquelle nous sommes arrivé lors de l'étude de la fondation du CERN. Cf. J. KRIGE, D. PeStre, " The How and the Why of the Birth of CERN ", History of CERN, op. cit. supra, n. 2, p. 523-544. 
le résultat est paradoxal. Il ne l'est pas tant si on admet une vision dynamique de ce qu'est un processus de décision complexe.

En guise de conclusion, nous aimerions revenir sur une dimension plus proprement historique et souligner ce qui, comparativement, nous semble constituer une certaine originalité de la physique des particules européenne. Dans la plupart des études consacrées à des décisions de gros équipements scientifiques et concernant l'Europe ou les États-Unis, apparaissent des équipes ou des laboratoires qui sont en position de concurrence directe vis-à-vis des gouvernements et qui cherchent à obtenir la plus large part d'une masse de crédits plus ou moins finie. Dans cette bataille, chaque groupe tend à définir un projet, projet sur lequel il parie et qui repose sur un type d'appareillage différent de celui des autres groupes (un accélérateur linéaire à électrons, un accélérateur à protons de type FFAG, etc.). Dès les premiers temps, chaque groupe cherche aussi à se trouver le maximum d'appuis dans et hors de la communauté scientifique, le plus grand nombre d'alliés possibles (ministres, hauts fonctionnaires, députés, etc.). La philosophie sous-jacente à ces attitudes est que tout est bon pour qui souhaite réussir, pour qui souhaite diriger un projet d'envergure et le voir financé. Ainsi en est-il dans le cas de la radio-astronomie française étudiée par Darmon et Lemaine, de la radio-astronomie britannique étudiée par Edge et Mulkay, dans le cas des projets franco-allemands des années 1960 étudiés par nous ou dans celui de la physique des hautes énergies américaines des années 1950 et 1960 (voir les travaux de Greenberg d'une part, de Westfall de l'autre ${ }^{61}$ ).

Dans le cas du CERN, les manières de faire semblent assez différentes : l'originalité réside dans l' "acceptation" par les grands patrons de la physique des hautes énergies européenne qu'il existe une sorte de hiérarchie des laboratoires, elle est dans la reconnaissance qu'une centralisation minimale est indispensable - elle est dans le refus d'une concurrence sauvage où tous les coups seraient permis. Cette hiérarchie, cette centralisation, se marque visuellement dans l'idée de la pyramide d'accélérateurs, pyramide européenne acceptée officiellement par l'ECFA en 1963 et qui doit être couronnée par la ou les deux machines européennes décidées collectivement, démocratiquement, oserait-on dire. En ce sens, pour le sommet de la pyramide, tout n'est pas permis; en particulier, on ne peut jouer librement une

61. Références données note 3. Le travail de Catherine WESTFALL sur le laboratoire de Fermi, près de Chicago, est en cours de rédaction. Ajouter D. PESTRE, "Année 1960 : un changement qualitatif dans les relations scientifiques franco-allemandes ", à paraître dans Revue d'Allemagne, 1988. 
machine nationale contre la machine européenne. En droit, chacun reste évidemment libre de procéder comme il l'entend chez lui et il ne faudrait pas idéaliser cette " manière " européenne des hautes énergies. Ici comme souvent, c'est nécessité qui fait vertu. Il n'empêche, le résultat est que, pour ce qui doit former la pointe avancée de la machine de guerre européenne, les scientifiques des divers pays " acceptent" de ne parler que d'une seule voix à leurs gouvernements.

Ceci permet de comprendre deux caractères bien marqués du processus que nous avons considéré dans la première partie de cette étude. D'abord, le fait qu'il est divisé en deux phases chronologiques successives, l'une pendant laquelle les scientifiques débattent seuls et parlent très peu d'argent - , l'autre pendant laquelle la vente collective aux gouvernements européens est organisée. Dans la première phase, on parle "physique " et on trouve beaucoup moins d'arguments d'autorité qu'aux États-Unis. Dans la seconde, on parle « tactique " - et ici le cynisme retrouve l'essentiel de ses droits. Dans cette phase, le médium essentiel est le Conseil du CERN, ensemble mixte formé pour moitié de scientifiques de haut rang, pour moitié de diplomates, mais animé d'un esprit de corps très fort et d'une loyauté sans faille à la physique des hautes énergies : il est le cœur tout-puissant et incontesté du dispositif européen contre lequel personne ne peut réellement se lever. À moins de se "suicider ", bien sûr : suffisamment puissant du côté scientifique et du côté politique, le Conseil pardonnerait difficilement à celui qui aurait mis en danger ce qui est au fond sa raison d'existence, les machines européennes les plus décisives, les plus prestigieuses, celles qui sont au sommet de la pyramide qu'il a comme fonction de faire s'édifier.

Cette situation assez originale de la physique des hautes énergies européenne conduit aussi à rappeler un autre trait. À la différence de ce que constatent beaucoup d'études à propos des estimations de coût, celles qui sont produites par le CERN apparaissent souvent assez fiables, proches de ce que sont ultérieurement les coûts réels (nous nous limitons ici aux coûts de construction des accélérateurs, les seuls qu'on puisse vérifier quelque peu). La raison - ou l'une des raisons premières - en est que, puisqu'il n'y a pas de concurrent sérieux, et qui plus est de concurrent qui pourrait masquer une part de ses propres coûts pour "emporter le marché ", l'intérêt bien compris de l'organisation est de jouer la carte de l'exactitude : à la différence de presque tous les autres qui trafiquent leurs chiffres et qui comptent sur le fait qu'on n'abandonne pas le financement d'un équipement dont la construction est assez avancée, les physiciens des hautes énergies sont 
gens sur qui on peut compter. Si l'on est sûr de pouvoir faire respecter la discipline - et le CERN y réussit - c'est la solution la plus intelligente pour la communauté dans son ensemble. Sur le long terme, elle est celle qui offre les meilleures relations avec les politiques.

Dominique PEstre, CNRS et CERN, Genève. 